Method A group of 2832, 1828, 2277 elderly residents aged $\geq 60$ in Beijing were chosen into this study in the year 2000, 2004, 2007 by well-established statistical sampling techniques such as cluster, stratification and random selection, and epidemiological trend of elderly hypertension was analysed by $\mathrm{x}^{2}$ analysis.

Result The prevalence rate $(69.2 \%, 61.9 \%, 56.0 \%)$ of hypertension and the control rate $(22.6 \%, 16.7 \%, 21.5 \%)$ lowered annually, and awareness rate $(43.7 \%, 55.8 \%, 57.6 \%)$ of treatment elevated annually. There was no rising in the control rate of male $(26.2 \%, 16.7 \%$, $20.8 \%)$, less older $(28.0 \%, 18.4 \%, 21.0 \%)$ and rural $(19.5 \%, 9.6 \%$, $13.4 \%$ ).

Conclusion The results indicate that the prevalence of hypertension is high in the elderly rural people, while the rates of awareness, treatment and control are low. It suggests that effective public measures need to be developed to improve the prevention and control of hypertension.

\section{e0244 HOW LOW WE SHOULD GO IN ELDERLY PATIENTS WITH TYPE 2 DIABETES AND HYPERTENSION IN CHINESE HAN PEOPLE}

doi:10.1136/hrt.2010.208967.244

Gang Chen, Xiaolan Lai, Lixiang Lin. Fujian Provincial Hospital, Fujian, China

Background The benefits of lowering systolic blood pressure (SBP) below $140 \mathrm{~mm} \mathrm{Hg}$ in elderly patients with type 2 diabetes and hypertension are unclear.

Methods Elderly (age $\geq 65$ years) patients with type 2 diabetes and hypertension underwent 12-lead ECG. The R-wave voltage in lead aVL $(\mathrm{RaVL} \geq 0.57 \mathrm{mV}$ ) was used to assess CVD risk. GFR was estimated by Cockcroft-Gault formula. 235 patients were tight SBP controlled (130-139 $\mathrm{mm} \mathrm{Hg}$ ), and 472 patients were less tight SBP controlled (140-159 mm Hg).

Results The height of $\mathrm{RaVL}$ and risk of the height of $\mathrm{RaVL}$ $\geq 0.57 \mathrm{mV}$ was no significance difference between the two groups, but some decline in eGFR was found in the less tight control group (adjusted mean 55.61 vs $59.65, \mathrm{p}=0.06$ ) although the decrease was not statistically significant.

Conclusions In elderly patients with type 2 diabetes and hypertension, to achieve the target SBP of below $140 \mathrm{~mm} \mathrm{Hg}$ seems reasonable.

\section{Q0245 A REPORT OF 511 INHOSPITAL CARDIOPULMONARY RESUSCITATION BASED ON THE UTSTEIN STYLE}

doi:10.1136/hrt.2010.208967.245

Song Wei, Chen Shi, Lan Baogiong, Mo Defana, He Ningninga.

Aim of the Study To use the Utstein Templates for Resuscitation Registries to evaluate the outcome and impact factors of cardiopulmonary resuscitation (CPR) at Hainan Provincial People's Hospital in China.

Material and Methods According to the "Utstein style" for CPR, a CPR registry form was designed and registry research methods were employed to study the epidemiological characteristics, outcome, and impact factors of CPR in 511 patients presented to the Emergency Department requiring CRP.

Results A total of 511 CPR patients were registered, including 356 males (69.7\%). The highest CPR rates were observed for 45-54, 55-64, and 65-74 year old patients. Cardiovascular diseases (190 cases, 36.5\%) and cerebrovascular diseases (48 cases, 9.2\%) were common in the medical histories of included patients. The ROSC and survival to discharge rates were $46.96 \%$ (139 patients) and $13.51 \%$ (40 patients) in patients treated only with in-hospital CPR but $16.74 \%$ (36 patients) and 4.65\% (10 patients), in CPR patients treated prior to presentation to the Emergency Department $(\mathrm{p}<0.01)$, respectively.

Conclusions These results are comparable with those of international horizontal studies using the "Utstein style." Nonetheless, the data collected in this study indicate that the survival to discharge rates for patients treated with in-hospital CPR are low and that the effectiveness of CPR needs to be improved.

\section{Q0246 THERAPEUTIC EFFECT OF A DUAL-CHAMBER PACEMAKER WITH THE OPTIMISED PROGRAM-CONTROL MODE ON LONG-OT SYNDROME}

doi:10.1136/hrt.2010.208967.246

Luo Yuanyuan, Sun Shao-Xi, Li Heng, Liu Fu-Qiang, Xu Ding-Li, Peng Jian. Nanfang Hospital, Southern Medical University, Guangzhou, China

Objective To explore the optimised program-control mode of a dualchamber pacemaker combined with $\beta$-blocker to treat congenital long QT syndrome (LOTS).

Methods 12 LQTS patients in our hospital that still have symptoms despite use of regular drug therapies or that can not endure the therapies were implanted with DDD cardiac pacemaker. The OT/ OTc intervals of those patients were measured at different pacing rates respectively. Their cardiac pacemakers were all programmed to selectively turn on and turn off some related functions at the pacing rate of 80 beats/min. The dosage of $\beta$-blockers was adjusted according to the patients' PR intervals and blood pressures. The MACE and the cardiac function of the patients were recorded after operation.

Results The measured OT/OTc interval decreased with the pacing rate increasing. The pacing rate of 80 beats/min can make QT/OTc interval basically normal. The MACE of the patients were statistically declined $(p=0.003)$ and no negative effect on cardiac function was found during the follow-up.

Conclusion The optimised program-control mode of a dual-chamber pacemaker combined with $\beta$-blocker to treat congenital LOTS are to pace at the rate of 80 beats/min and program to turn off lag, sleep, automatic preventing PMT and automatic threshold-capture feature and turn on the PVC, rate adaptation and atrioventricular node priority function.

\section{e0247 PREVALENCE OF METABOLIC SYNDROME AND ITS EFFECT ON CAROTID ARTERY INTIMA-MEDIA THICKNESS IN XINJIANG KAZAK POPULATIONS}

doi:10.1136/hrt.2010.208967.247

Zixiang Yu, Yitong Ma, Yining Yang, Ding Huang, Xiang Ma, Zhenyan Fu. First affiliated Hospital, Xinjiang Medical University, China

Objective To investigate the prevalence of metabolic syndrome (MS) in Xinjiang Kazak populations, and explore the effect of metabolic syndrome on Carotid artery intima-media thickness in Kazak populations.

Methods A cross sectional study was conducted in 1610 kazak participants aged from 19 years to 98 years (mean \pm SD, $46.7 \pm 12$; 654 men and 956 women) in Xinjiang Yili. The National Cholesterol Education Program (NCEP) criteria for MS were used in the study. Carotid intima-media thickness (IMT) was measured by echocardiography. According to NCEP criteria, populations were divided into MS group and non-MS group.

Results The prevalence rate of MS by the NCEP criteria Was $40.1 \%$ (44.8\% in men and $36.9 \%$ in women). IMT was significantly higher in $\mathrm{MS}$ group than non-MS group $(\mathrm{p}<0.05)$. 